\title{
Green Synthesis of Copper Nano-Drug and Its Dental Application upon Periodontal Disease-Causing Microorganisms
}

\author{
Sanaa M. F. Gad El-Rab ${ }^{1,2 *}$, Sakeenabi Basha ${ }^{3}$, Amal A. Ashour ${ }^{4}$, Enas Tawfik Enan ${ }^{5,6}$, \\ Amal Ahmed Alyamani ${ }^{1}$, and Nayef H. Felemban ${ }^{7}$ \\ ${ }^{1}$ Department of Biotechnology, Faculty of Science, Taif University, P.O. Box 888, Taif 21974, KSA \\ ${ }^{2}$ Department of Botany and Microbiology, Faculty of Science, Assiut University, Assiut 71516, Egypt \\ ${ }^{3}$ Department of Preventive and Community Dentistry, Faculty of Dentistry, Taif University, Taif 26571, Saudi Arabia \\ ${ }^{4}$ Department of Oral and Maxillofacial Surgery and Diagnostic Sciences, Oral Pathology Division, Faculty of \\ Dentistry, Taif University, Taif 21431, Saudi Arabia \\ ${ }^{5}$ Dental Biomaterials, Faculty of Dentistry, Taif University, Taif 26571, Saudi Arabia \\ ${ }^{6}$ Dental Biomaterials, Faculty of Dentistry, Mansoura University, Dakahleya 35516, Egypt \\ ${ }^{7}$ Preventive Dentistry Department, Faculty of Dentistry, Taif University, Taif 26571, Saudi Arabia
}

\begin{abstract}
Dental pathogens lead to chronic diseases like periodontitis, which causes loss of teeth. Here, we examined the plausible antibacterial efficacy of copper nanoparticles (CuNPs) synthesized using Cupressus macrocarpa extract (CME) against periodontitis-causing bacteria. The antimicrobial properties of CME-CuNPs were then assessed against oral microbes (M. luteus. B. subtilis, $P$. aerioginosa) that cause periodontal disease and were identified using morphological/ biochemical analysis, and 16S-rRNA techniques. The CME-CuNPs were characterized, and accordingly, the peak found at $577 \mathrm{~nm}$ using UV-Vis spectrometer showed the formation of stable CME-CuNPs. Also, the results revealed the formation of spherical and oblong monodispersed CME-CuNPs with sizes ranged from 11.3 to $22.4 \mathrm{~nm}$. The FTIR analysis suggested that the CME contains reducing agents that consequently had a role in Cu reduction and CME-CuNP formation. Furthermore, the CME-CuNPs exhibited potent antimicrobial efficacy against different isolates which was superior to the reported values in literature. The antibacterial efficacy of CME-CuNPs on oral bacteria was compared to the synergistic solution of clindamycin with CME-CuNPs. The solution exhibited a superior capacity to prevent bacterial growth. Minimum inhibitory concentration (MIC), minimum bactericidal concentration (MBC), and fractional inhibitory concentration (FIC) of CME-CuNPs with clindamycin recorded against the selected periodontal disease-causing microorganisms were observed between the range of $2.6-3.6 \mu \mathrm{g} / \mathrm{ml}, 4-5 \mu \mathrm{g} / \mathrm{ml}$ and $0.312-0.5$, respectively. Finally, the synergistic antimicrobial efficacy exhibited by CME-CuNPs with clindamycin against the tested strains could be useful for the future development of more effective treatments to control dental diseases.
\end{abstract}

Received: June 4, 2021 Accepted: September 6, 2021 First published online: September 8, 2021

*Corresponding author Phone: +00201025475454 E-mail: sanaafahmy@aun.edu.eg

pISSN 1017-7825 eISSN 1738-8872

Copyright $@ 2021$ by the authors. Licensee KMB. This article is an open access article distributed under the terms and conditions of the Creative Commons Attribution (CC BY) license.
Keywords: Antibiotic, antimicrobial activity, copper nanoparticles, periodontal disease

\section{Introduction}

As of 2017, it was estimated that about 3.5 billion people have dental diseases such as tooth caries and periodontitis infections in their permanent teeth [1]. Periodontal disease is one of the major public health problems in many countries [2,3]. It presents as a chronic, inflammatory, and infectious disease that affects the teeth and surrounding structures $[4,5]$, causing in the most severe cases, excessive gingival bleeding and inflammation, increased dental mobility, and finally, tooth loss [6]. Studies have shown that disturbance of microbiota may activate P. aeruginosa, E. coli, S. pyogenes, and B. cereus [7-9]. In the periodontal site of chronic patients, high distribution of B. cereus, S. pyogenes, P. aeruginosa, and E. coli commonly occurs [10]. Research has also suggested periodontal disease as the most common oral infectious disease that is predominated by microorganisms [11]. Also, bacterial resistance in biofilms to anti-microbial treatment can be up to 1,000 times greater than that of planktonic organisms [12] which presents a major global health problem [13] and causes tooth loss $[14,15]$. Clindamycin is an antibiotic that is usually advised as effective therapy because of its excellent coverage of typical oral flora [16]. Currently, microbial resistance to conventional antibiotics is considered an important health problem and has raised the demand for more effective solutions [17]. The discovery of novel, strategic and effective antimicrobial agents to prevent microbial infections is needed [18] to prevent plaque 
formation and maturation as the first line of therapy [19]. The study of synergistic effects of metal nanoparticles with antibiotics and their antimicrobial effect on periodontal disease-causing microorganisms is an important aim.

Copper nanoparticles (CuNPs) exhibit high antimicrobial activity against different species of microorganisms including fungi, gram-negative and gram-positive bacteria; however, there have been no reports on their activity against dental pathogens. In antimicrobial therapies, the use of CuNPs would require and depend on therapeutic time and concentration [20]. Eco-friendly materials such as microbes, plants, and enzymes are used for the biosynthesis of CuNPs because they are nontoxic materials as well as being simple to use and low cost [21-23].

In this study, we adopted dental prophylaxis as our objective and sought to synthesize antibacterial nano copper using an herbal formulation of CME as a reducing agent against periodontal infectious disease-causing microorganisms such as gram-positive B. subtilis, M. luteus, in addition to gram-negative P. aeruginosa. We also evaluated the antimicrobial efficiency of combining antibiotics and biosynthesized CuNPs to overcome antibiotic resistance.

\section{Materials and Methods \\ Preparation of Cupressus macrocarpa Aqueous Extract}

Cupressus macrocarpa plant was identified by Prof. Ahmed A. El-Settawy (Head of Forestry and Wood Technology Department at the Faculty of Agriculture, Alexandria University) and given the voucher number Zidan 313 [24]. Aqueous extract of Cupressus macrocarpa leaves was prepared by taking $25 \mathrm{~g}$ of leaves, rinsing it in a glass beaker containing deionized water $(100 \mathrm{ml})$, and heating at $85^{\circ} \mathrm{C}$ for $30 \mathrm{~min}$ and then filtering and storing it for subsequent use [25].

\section{Biosynthesis of CuNPs}

Forty milliliters of copper acetate (Sigma-Aldrich, USA) and then $10 \mathrm{ml}$ of CME were added in an Erlenmeyer flask. Then, $10 \mathrm{ml}$ of the CME was added. The mixture was irradiated in a microwave oven (Matsushita Electric Industrial Co., Ltd. Panasonic, Japan) at $800 \mathrm{~W}$ for $1 \mathrm{~h}$ until the formation of copper nanoparticles (CME-CuNPs), and allowed to cool at room temperature [26].

\section{Characterization of CME-CuNPs}

Characterization of CME-CuNPs was done using the following methods:

UV-Visible spectrum: UV-vis spectrometer (Shimadzu UV-1650) was used to record absorbance in the range of 300-700 $\mathrm{nm}$ and monitor the rate of CME-CuNP formation [27].

Transmission electron microscopy (TEM): TEM analysis was done using the TEM JEOL $100 \mathrm{kV}$ (Assiut Electron Microscope Unit). The CME-CuNPs were prepared by placing a drop of the suspension on carbon-coated copper grids and allowing it to dry on the grid for $4 \mathrm{~min}$. The shape and size of CME-CuNPs were determined from the TEM micrograph [28].

\section{X-Ray Diffraction Analysis}

The nature and size of the CME-CuNPs were determined using Shimadzu XRD (Shimadzu XD-3A, Japan). The nanoparticle size was calculated using the formula of Debye-Scherrer [29].

Fourier-transform infrared analysis (FTIR): The spectra of CME and CME-CuNPs were determined using a Shimadzu IR-470 Spectrometer (Shimadzu) in the range of 500-4,000 $\mathrm{cm}^{-1}[30]$.

\section{Isolation and Identification of Bacterial Isolates}

Ethical approval for the current study (Ref. No. 41-1107-00152) was provided by the Ethics Committee of Research, Taif University, Taif, KSA. Bacterial isolates were obtained from the Faculty of Dentistry Medical Diagnosis and Infection Control Unit, Taif University, Taif, KSA. Isolation of bacteria collected from the mouths of patients (dental pulp, and dental plaque) was conducted by provisional researchers from patients infected with periodontal disease-causing microorganisms using sterile root canal instruments and the isolates were stored in sterile saline in a $10 \mathrm{ml}$ saline kit. One hundred isolates were isolated on Mueller-Hinton agar (Thermo Scientific Oxoid), for $24 \mathrm{~h}$ at $37^{\circ} \mathrm{C}$ under aerobic condition and suspected single colonies were then identified by morphological/biochemical tests according to the criteria $[31,32]$.

\section{Molecular Characterization Using 16S rRNA Gene}

Bacterial cells were used for collecting genomic DNA with the Wizard Genomic DNA Purification Kit (Promega, USA) and used as a PCR template. In $20 \mu \mathrm{l}$ of the polymerase chain reaction (PCR) reaction solution containing forward primer $27 \mathrm{~F}$ and reverse primer $1492 \mathrm{R}$, one microliter of DNA template was added. Then, 35 amplification cycles of $16 \mathrm{~S} \mathrm{rRNA}$ genes were performed at $94^{\circ} \mathrm{C}$ for $45 \mathrm{~s}$ in the denaturation step, at $55^{\circ} \mathrm{C}$ for $60 \mathrm{~s}$ in the annealing step, and then at $72^{\circ} \mathrm{C}$ for $60 \mathrm{~s}$ in the extension step. DNA fragments were amplified up to $1,400 \mathrm{bp}$. The products of $16 \mathrm{~S}$ rRNA of approximately $1,400 \mathrm{bp}$ were sequenced using $518 \mathrm{~F} / 800 \mathrm{R}$ primer set. The sequencing of $16 \mathrm{~S}$ rRNA has been analyzed on an automated Applied BioSystems, DNA sequencing system, (model 3730XL, USA). The similarity of bacterial strains was aligned using CLUSTAL W (1.81) phylogenetic tree obtained from the nucleotide sequence databases.

\section{Antibacterial Activity of CME-CuNPs}

Micro-Dilution Test for Determination of MIC and MBC

The MBC of CME-CuNPs, clindamycin, or both is defined as the lowest concentration of an antimicrobial 
agent killing the majority (99.99\%) of bacterial inoculums. Since the MIC of CME-CuNPs, clindamycin, or both relates to their inhibitory ability, it is possible that if the antimicrobial agent were removed, the bacteria would begin to grow again [33]. The test was performed with serial dilutions of clindamycin (from $64 \mu \mathrm{g} / \mathrm{ml}$ to $8 \mu \mathrm{g} / \mathrm{ml}$ ), CME-CuNPs (from $27 \mu \mathrm{g} / \mathrm{ml}$ to $9 \mu \mathrm{g} / \mathrm{ml}$ ) or clindamycin with CME-CuNPs (from $5 \mu \mathrm{g} / \mathrm{ml}$ to $2.6 \mu \mathrm{g} / \mathrm{ml}$ ) arranged across the rows in Mueller-Hinton broth and inoculation of the wells of a micro-dilution plate with the bacterial culture. The MIC and MBC of CME-CuNPs, clindamycin, or both were determined against the tested isolates [34]. All samples of clindamycin, CME-CuNPs, or CME-CuNPs with clindamycin $(1 \mu \mathrm{g} / \mathrm{ml}: 1 \mu \mathrm{g} / \mathrm{ml})$ were tested in triplicate, and the test was repeated five separate times.

\section{Antibacterial Synergy Test}

The antibacterial synergy in vitro test was carried out using a checkerboard synergy method based on the value of the FIC (fractional inhibitory concentration). The FIC value was calculated by comparing the MIC of each agent CME-CuNP and clindamycin with the MIC of the CME-CuNP and clindamycin combination. Clindamycin was added in concentrations between 64 and $8 \mu \mathrm{g} / \mathrm{ml}$ and CME-CuNPs in a range of 10-30 $\mu \mathrm{g} / \mathrm{ml}$. These effects can be quantified by the application of mathematical expressions: the fractional inhibitory concentration (FIC) of combinations among CME-CuNPs and clindamycin against tested strains. For two antibacterial agents, CME-CuNPs (A) and clindamycin (B) acting individually or in combination:

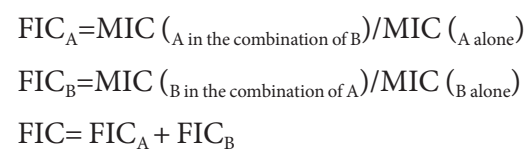

An FIC index of $<0.5$ indicates synergism, $>0.5-1$ indicates additive effects, $>1$ to $<2$ indifference, and $\geq 2$ is considered to be antagonism [34].

\section{Statistical Analysis}

Descriptive summary statistics were obtained for all independent and outcome variables. The mean difference was tested using analysis of variance (ANOVA) followed by Tukey's post hoc and $t$-test. The analysis was obtained using the Statistical Package for Social Science version 17 (SPSS INC Chicago link). All statistical tests were twosided, and the significance level was set at $p<0.05$.

\section{Results}

Biosynthesis of CME-CuNPs

In the current research, CME-CuNPs were biosynthesized by reducing copper acetate to CME-CuNPs using $\mathrm{CME}$. The formation of CME-CuNPs in the reaction mixture is indicated by a change in the color from blue to brown.

\section{Characterization of CME-CuNPs}

UV-Vis spectroscopy of CME-CuNPs: UV-Vis absorption was studied after the formation of CME-CuNPs was dispersed and absorbance was measured by using a UV-visible spectrophotometer between 450 and $800 \mathrm{~nm}$. CME-CuNPs showed peaks at $577 \mathrm{~nm}$ as shown in Fig. 1. The UV-visible peak at $577 \mathrm{~nm}$ was for pure CMECuNPs.

\section{Transmission Electron Microscope Analysis}

The CME-CuNPs were characterized by TEM analysis (Fig. 2). The average size of the CME-CuNPs was estimated to be at 11.3-22.4 nm, via TEM analysis. TEM analysis of the CME-CuNPs showed spherical to oblong, polydispersed shapes (Fig. 2).

\section{X-Ray Diffraction (XRD)}

X-ray diffraction (XRD) pattern showed four major peaks at $2 \theta$ values of $47^{\circ}, 54^{\circ}$, and $72^{\circ}$. These characteristic peaks could be attributed to reflection planes (111), (200), and (220) of the face-centered cubic crystalline (FCC) structure of pure metallic copper (Fig. 3).

\section{Comparison of FTIR Spectra of Cupressus macrocarpa Extract, CME-CuNPs}

Fourier-transform infrared analysis (FTIR) was used to characterize the CME and the resulting CME-CuNPs. Absorbance bands were observed in the region 500 to $4,000 \mathrm{~cm}^{-1}$, (Figs. 4A and 4B). Fig. 4A showed the absorption bands of plant extract. There is a broad and strong band in the region $3,429 \mathrm{~cm}^{-1}$ ascribed to $\mathrm{O}-\mathrm{H}$ stretching vibrations. According to a known standard, a band in the $2,920 \mathrm{~cm}^{-1}$ region originates from $\mathrm{C}-\mathrm{H}$ (hydrocarbon) stretching vibration, $1,631 \mathrm{~cm}^{-1}$, denoting an amine group related to proteins, $1,411 \mathrm{~cm}^{-1}$, representing methylene - $\mathrm{CH}$ bending bond, and $1,094 \mathrm{~cm}^{-1}$ of primary alcohol/C-O- stretching. The CME caused a reduction of copper ions. Fig. 4B showed the absorption bands of CME-CuNPs. Peaks at 3,402, 2,927, 1,631, 1,404, and 1,060 $\mathrm{cm}^{-1}$ represent the $\mathrm{OH}$ functional groups, an amine group $(\mathrm{NH})$, stretching $-\mathrm{CH}$, and $\mathrm{C}-\mathrm{O}$ stretching vibrations, respectively. Polyphenolics, proteins, amino acids, and carbohydrate compounds were adsorbed on the surface of the CME-CuNPs. 
A

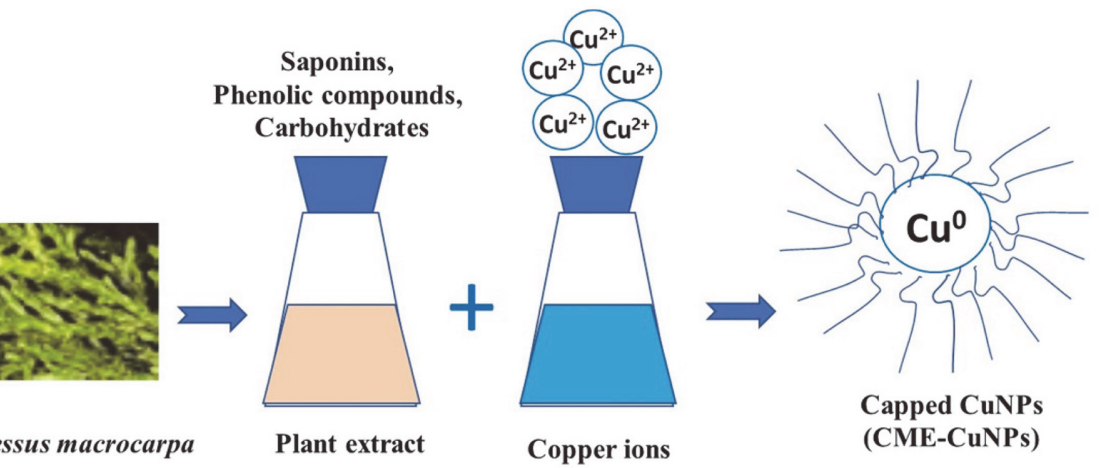

Cupressus macrocarpa

B

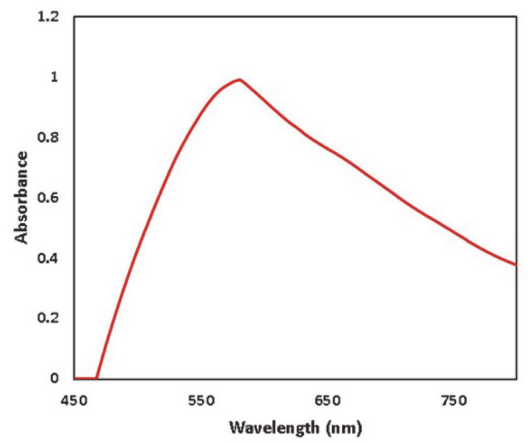

Fig. 1. (A) Illustration showing biosynthesis of CME-CuNPs. Notes: Photograph showing the formation of CME-CuNPs using Cupressus macrocarpa extract. (B) UV-visible spectrum of CME-CuNPs.

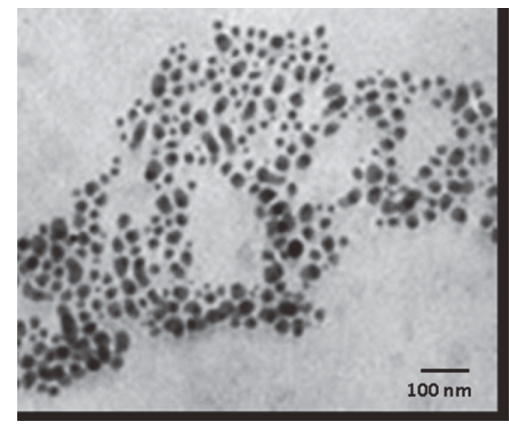

Fig. 2. TEM images of the synthesized CME-CuNPs.

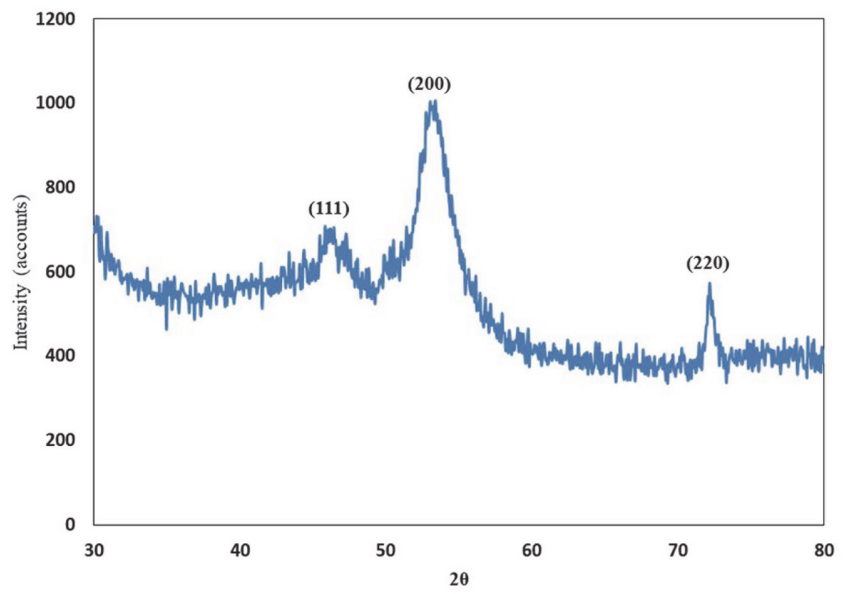

Fig. 3. XRD patterns of the synthesized CME-CuNPs with aqueous extract of Cupressus macrocarpa. 


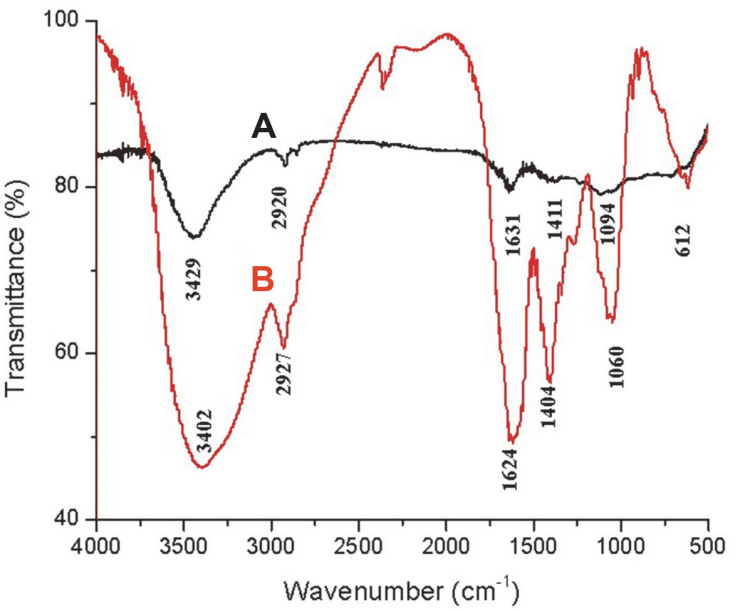

Fig. 4. FTIR spectra of Cupressus macrocarpa extract (A) and the synthesized CME-CuNPs (B).

Table 1. Morphological and biochemical characteristics of bacteria strains (MIC1, BAC1 and PSE5).

\begin{tabular}{|c|c|c|c|c|c|c|c|c|c|c|c|c|c|c|c|c|c|c|c|c|}
\hline \multirow{3}{*}{$\begin{array}{c}\text { Bacterial } \\
\text { isolates }\end{array}$} & \multicolumn{19}{|c|}{ Characteristics } & \multirow[b]{3}{*}{ Probable bacteria } \\
\hline & \multirow{2}{*}{ 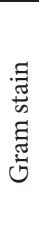 } & \multicolumn{2}{|c|}{$\begin{array}{c}\text { Cell } \\
\text { morphology }\end{array}$} & \multirow{2}{*}{ 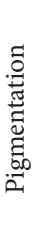 } & \multirow{2}{*}{ 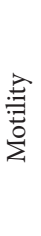 } & \multirow{2}{*}{ 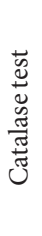 } & \multirow{2}{*}{ 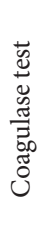 } & \multirow{2}{*}{ 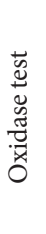 } & \multirow{2}{*}{ 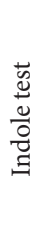 } & \multirow{2}{*}{ 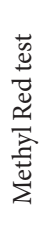 } & \multirow{2}{*}{ 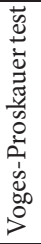 } & \multirow{2}{*}{ 苛 } & \multirow{2}{*}{ 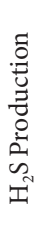 } & \multirow{2}{*}{ 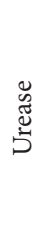 } & \multirow{2}{*}{ 䓠 } & \multicolumn{4}{|c|}{ Fermentation } & \\
\hline & & $\frac{n}{0}$ & ت্তু & & & & & & & & & & & & & $\frac{\ddot{\infty}}{\stackrel{0}{0}}$ & 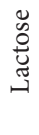 & 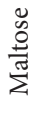 & 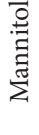 & \\
\hline MIC1 & + & - & + & + & - & + & - & + & - & - & - & - & - & + & + & - & - & - & - & Micrococcus luteus \\
\hline $\mathrm{BAC1}$ & + & + & - & - & + & + & + & - & - & - & + & + & - & - & + & + & + & + & + & Bacillus subtilis \\
\hline PSE5 & - & + & - & + & + & + & - & + & - & - & - & + & - & - & + & + & - & + & + & Pseudomona aeruginosa \\
\hline
\end{tabular}

(-): Negative, (+): Positive

Isolation and Identification of Drug-Resistant Bacteria

One hundred isolates were collected on Mueller-Hinton agar at $37^{\circ} \mathrm{C}$. Then, the suspected single colonies were identified as M. luteus (50\%), B. subtilis (12.5\%), and P. aeruginosa (12.5\%) by morphological/biochemical tests according to the criteria.

The strains were morphologically and biochemically characterized as M. luteus, B. subtilis, and P. aeruginosa and designated as M. luteus MIC1, B. subtilis BAC1, and P. aeruginosa PSE1, respectively. The aerobic strains MIC1, ABC1, and PSE5 were chosen for the current study (Table 1).

\section{S rRNA Analysis}

Partial 16S rRNA gene sequences were used to identify multidrug-resistant bacterial isolates. MIC1, BAC1, and PSE5 were carried and sequenced. The 16S rRNA gene sequences of the bacterial isolates from the dental pulp and plaque were deposited in the DDBJ/EMBL/GenBank nucleotide sequence databases with the following accession numbers (Table 2): MIC1 strain (LC628029), BAC1 strain (LC628030), and PSE5 strain (LC628031). The 16S rRNA gene sequences of strains were found to be 1310, 1240, and $1312 \mathrm{nt}$ in length, for strain MIC1, BAC1, and PSE5, respectively.

A dendrogram demonstrating the results of the 16S rRNA of M. luteus (MIC1), B. subtilis (BAC1), and P. aeruginosa (PSE5) analysis is displayed in Figs. 5A, 5B, 5C. The results show the highest similarity of isolates MIC1, BAC1, and PSE5 to members of the Micrococcus, Bacillus, and Pseudomonas group, respectively. Moreover, the 16S rRNA sequences of the isolates MIC1, BAC1, and PSE5 are the most closely associated with M. luteus, B. subtilis, and P. aeruginosa (Table 2). The 16S rRNA gene of MIC1, BAC1, and PSE5 displayed 98.31\%, 96.43\%, and 97.22 similarities to Micrococcus luteus DSM 20030 (NR037113.1), Bacillus subtilis subsp. subtilis 168 (NR102783.2), and Pseudomonas aeruginosa ATCC 10145 (NR114471.1), respectively.

Table 2. The bacterial isolates (MIC1, BAC1 and PSE5) of oral infections.

\begin{tabular}{ccc}
\hline Bacterial isolates & Accession No. & Closest neighbor \\
\hline MIC1 & LC628029 & M. luteus \\
BAC1 & LC628030 & B. subtilis \\
PSE5 & LC628031 & P. aeruginosa \\
\hline
\end{tabular}




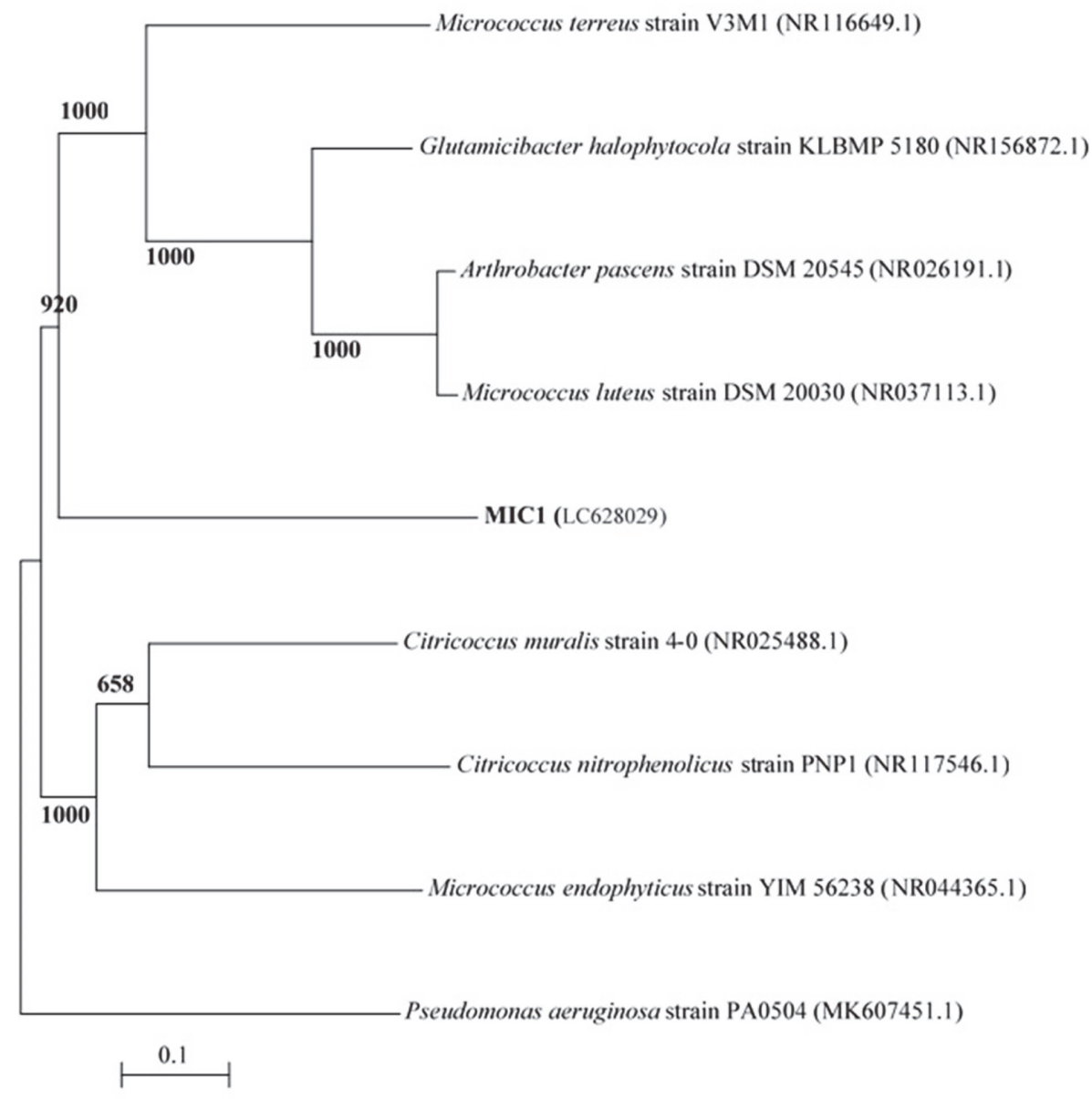

Fig. 5A. A phylogenetic tree of bacterial isolates relied on the nucleotide sequences of $16 \mathrm{~S}$ rRNA genes, constructed by neighbor-joining method. The scale bar displays the genetic distance. The number presented next to each node displays the percentage bootstrap value of 1000 replicates. The Pseudomonas aeruginosa strain PA0504 was treated as the out-group. The GenBank accession numbers of the bacteria are presented in parentheses.

\section{Antimicrobial Activity}

Minimum Inhibitory Concentration (MIC) and Minimum Bactericidal Concentration (MBC) Assays

In this study, three strains resistant to clindamycin were used. The MIC of CME-CuNPs was found to be 13.5, 18 , and $18 \mu \mathrm{g} / \mathrm{ml}$ against $M$. luteus, B. subtilis, and P. aeruginosa, respectively (Table 3 ), while the MIC of CMECuNPs with clindamycin was found to be 2.6,3.6, and $3.6 \mu \mathrm{g} / \mathrm{ml}$, respectively. Moreover, the MBC of CME-CuNPs was determined to be 20,27 , and $27 \mu \mathrm{g} / \mathrm{ml}$ for M. luteus, B. subtilis, and P. aeruginosa, respectively, while the MBC of CME-CuNPs with clindamycin was determined to be 4,5 , and $5 \mu \mathrm{g} / \mathrm{ml}$, respectively. The MICs of CME-CuNPs with clindamycin significantly decreased by more than 5 folds compared to CME-CuNPs alone while it decreased by more than 3-10 folds compared to clindamycin alone. Our data showed that CME-CuNPs have a small size with high antimicrobial activity. As shown in Table 4, MICs of CME-CuNPs were the lowest concentration (13.5-18 $\mu \mathrm{g} / \mathrm{ml}$ ) compared to MICs of CuNPs in earlier studies $(40 \mathrm{mg} / \mathrm{ml}, 75 \mu \mathrm{g} / \mathrm{ml}, 100 \mu \mathrm{g} / \mathrm{ml}$, and 150-225 $\mu \mathrm{g} / \mathrm{ml}$ ) on antibacterial activity against bacteria.

Table 3. MIC, MBC and of CME-CuNPs and CME-CuNPs with clindamycin $(\mu \mathrm{g} / \mathrm{ml})$ against oral bacteria in Mueller Hinton broth.

\begin{tabular}{lccccccccc}
\hline \multirow{2}{*}{ Isolate } & \multicolumn{3}{c}{ Clindamycin $(\mu \mathrm{g} / \mathrm{ml})$} & \multicolumn{2}{c}{ CME-CuNPs $(\mu \mathrm{g} / \mathrm{ml})$} & \multicolumn{3}{c}{ CME-CuNPs with clindamycin $(\mu \mathrm{g} / \mathrm{ml})$} \\
\cline { 2 - 9 } & $\mathrm{MIC}$ & $\mathrm{MBC}$ & $\mathrm{MBC} / \mathrm{MIC}$ & $\mathrm{MIC}$ & $\mathrm{MBC}$ & $\mathrm{MBC} / \mathrm{MIC}$ & $\mathrm{MIC}$ & $\mathrm{MBC}$ & $\mathrm{MBC} / \mathrm{MIC}$ \\
\hline M. luteus MIC1 & 8 & 16 & 2 & 13.5 & 20 & 1.5 & 2.6 & 4 & 1.5 \\
B. subtilis BAC1 & 32 & 64 & 2 & 18 & 27 & 1.5 & 3.6 & 5 & 1.4 \\
P. aeruginosa PSE1 & 32 & 64 & 2 & 18 & 27 & 1.5 & 3.6 & 5 & 1.4 \\
\hline
\end{tabular}

MIC: Minimum inhibitory concentration, MBC: Minimum bactericidal concentration. 


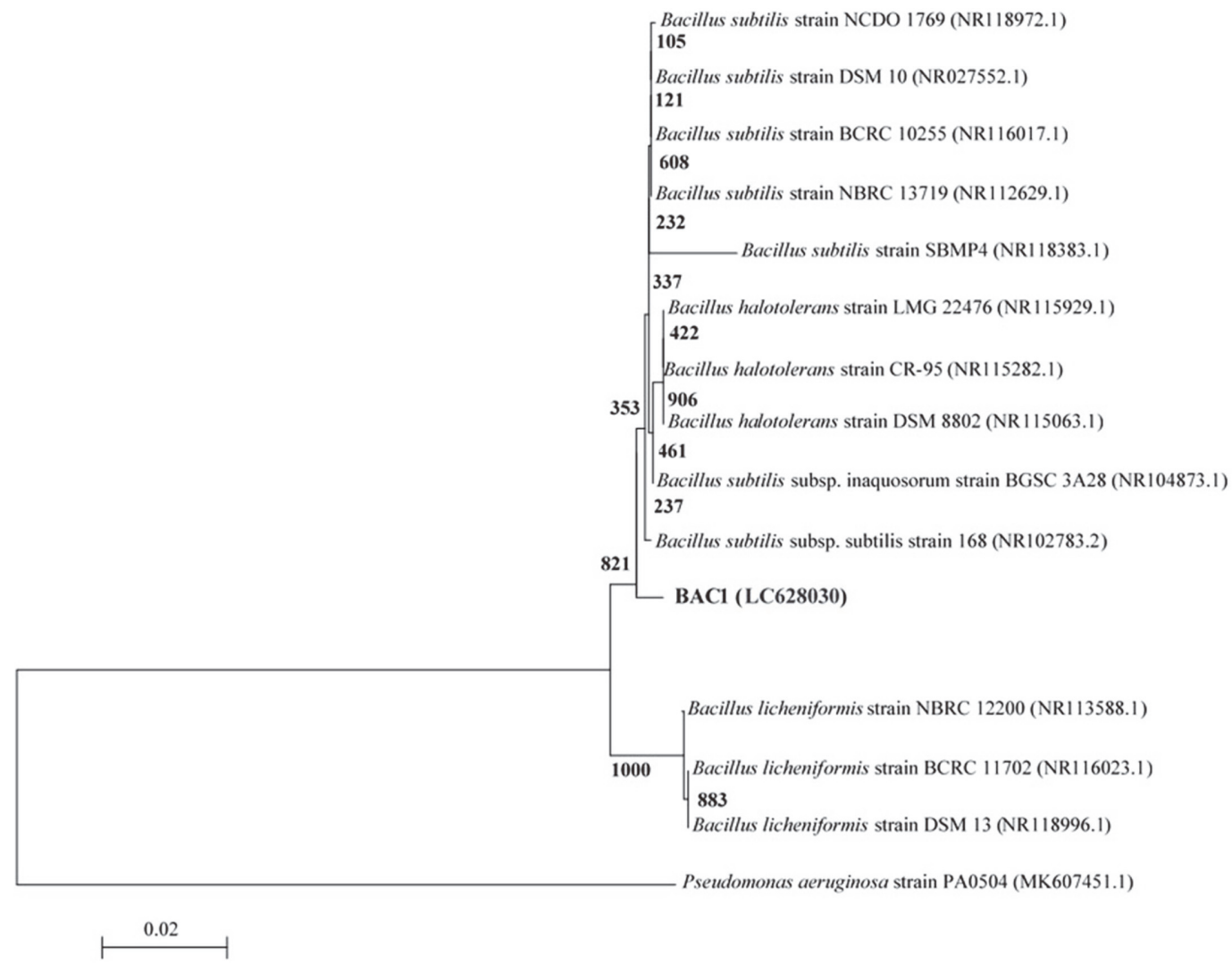

Fig. 5B. A phylogenetic tree of bacterial isolates relied on the nucleotide sequences of 16S rRNA genes, constructed by neighbor-joining method. The scale bar displays the genetic distance. The number presented next to each node displays the percentage bootstrap value of 1000 replicates. The Pseudomonas aeruginosa strain PA0504 was treated as the out-group. The GenBank accession numbers of the bacteria are presented in parentheses.

Table 4. Comparison of antibacterial activity for CME-CuNPs with earlier studies.

\begin{tabular}{llllr}
\hline \multicolumn{1}{c}{ Materials } & \multicolumn{1}{c}{ Size $(\mathrm{nm})$} & $\begin{array}{c}\text { Concentrations } \\
(\mu \mathrm{g} / \mathrm{ml})\end{array}$ & Bacteria & References \\
\hline CuNPs & $55-350 \mathrm{~nm}$ & $40 \mathrm{mg} / \mathrm{disc}$ & Staphylococcus aureus (S. aureus), Escherichia coli (E. coli) & {$[50]$} \\
CuNPs & 4.7 to $17.4 \mathrm{~nm}$ & $100 \mu \mathrm{g} / \mathrm{ml}$ & Bacillus, S. aureus, E. coli, and P. aeruginosa & {$[51]$} \\
CuNPs & $60-90 \mathrm{~nm}$ & $75 \mu \mathrm{g} / \mathrm{ml}$ & Streptococcus sp. E. coli & {$[52]$} \\
CuNPs & $131 \mathrm{~nm}$ & $40 \mu \mathrm{g} / \mathrm{ml}$ & Streptococcus mutans & {$[53]$} \\
CuNPs & $80 \mathrm{~nm}$ & $150-225 \mu \mathrm{g} / \mathrm{ml}$ & Enterococcus faecalis & {$[54]$} \\
CME-CuNPs & $11.3-22.4 \mathrm{~nm}$ & $13.5-18 \mu \mathrm{g} / \mathrm{ml}$ & B. subtilis, M. luteus, P. aeruginosa & Present study \\
\hline
\end{tabular}

Table 5. FIC index of combinations among CME-CuNPs and clindamycin against tested strains.

\begin{tabular}{ccc}
\hline Bacterial isolates & FIC index \\
\hline MIC1 & 0.500 & $(\mathrm{~S})$ \\
BAC1 & 0.312 & $(\mathrm{~S})$ \\
PSE5 & 0.312 & (S) \\
\hline
\end{tabular}

An FIC index of $<0.5$ indicates synergism (S), $>0.5-1$ indicates additive effects (AD), $>1$ to $<2$ indifference (ID), and $\geq 2$ is considered to be antagonism (AN).

Antibacterial Synergy Test

The FIC of CME-CuNPs and the clindamycin combination were investigated and are summarized in Table 5. This experiment showed that CME-CuNPs acted synergistically $(p<0.5)$ with clindamycin against B. subtilis, M. luteus, in addition to $P$. aeruginosa. 


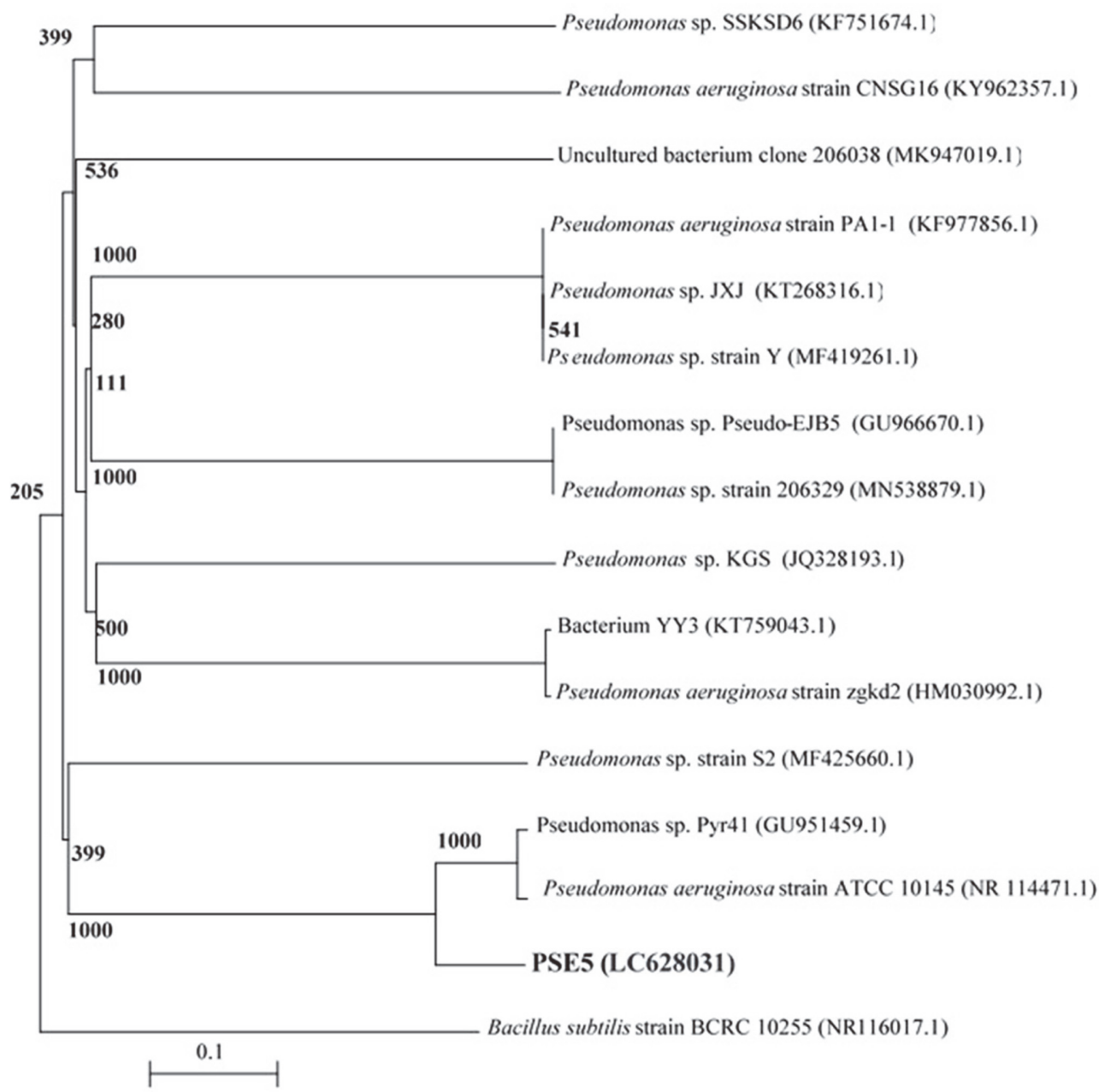

Fig. 5C. A phylogenetic tree of bacterial isolates relied on the nucleotide sequences of 16S rRNA genes, constructed by neighbor-joining method. The scale bar displays the genetic distance. The number presented next to each node displays the percentage bootstrap value of 1000 replicates. The Bacillus subtilis strain BCRC 10255 was treated as the out-group. The GenBank accession numbers of the bacteria are presented in parentheses.

A

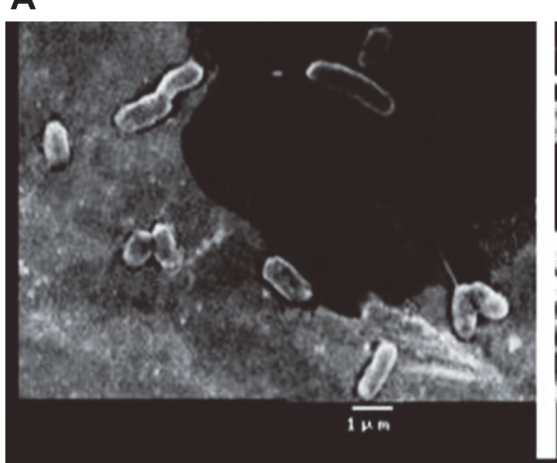

B

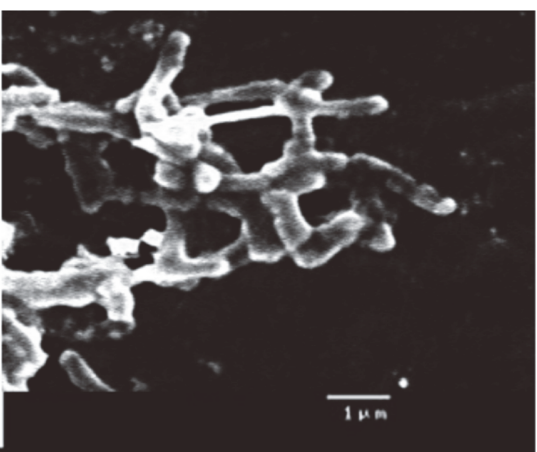

Fig. 6. SEM observation of control B. subtilis (a) and images shows the CME-CuNP treated B. subtilis (b) showing membrane damage, and cell elongation in treated cells.

\section{Mechanism of Action}

The CME-CuNP treated B. subtilis was observed with distorted membrane morphology, and cell elongation, (Fig. 6B) while the untreated control B. subtilis cells were found to be compact, tightly packed cells (Fig. 6A). In our results, the bacteria cells were distorted and CME-CuNPs act as an inhibitor for selected bacteria. 


\section{Discussion}

In this study, we applied a combination of CME-CuNPs and clindamycin. When CME-CuNPs with clindamycin antibacterial properties were compared with CME-CuNPs alone against tested bacteria, CME$\mathrm{CuNPs}$ with clindamycin proved to be stronger. Based on our results, the present study confirmed the success of the biosynthesis of CME-CuNPs from CME. The CME-CuNPs biosynthesis reaction progress was monitored for color change and UV-Vis spectrometric analysis. The change of color from blue to brown in the reaction mixture forming CME-CuNPs agreed with $\mathrm{Wu}$ et al. (2020) [35] who similarly observed the color change during the synthesis of CuNPs. The absorbance peaks recorded at $577 \mathrm{~nm}$ are specific to CME-CuNPs and were attributed to the surface plasmon resonance phenomenon which indicates the formation of CME-CuNPs. Various studies have also recorded similar CuNPs SPR peaks $[26,36,37]$. TEM images of nano copper: the appearance is spherical to oblong with a size of about 11.3-22.4 nm [38]. There are three lattice planes that are (111), (200), and (220) of the face-centered cubic (fcc) structure that was observed for CME-CuNPs at De Bragg's reflection angles of $47^{\circ}, 54^{\circ}$, and $72^{\circ}$, respectively. No other impurity peak was detected in the CME-CuNP sample. The XRD results reflected the purity of CME-CuNPs in the present study and this is in accordance with previous studies $[39,40]$.

The organic compounds in the CME reduced and stabilized the CME-CuNPs, thereby preventing agglomeration. The CME contains FTIR peaks at 3,402, 2,927, 1,631, 1,404, and 1,060 $\mathrm{cm}^{-1}$ representing the $\mathrm{OH}$ functional groups, an amine group $(\mathrm{NH})$, stretching $-\mathrm{CH}$, and $\mathrm{C}-\mathrm{O}$ stretching vibrations, respectively. These functional groups of the CME related to polyphenolics, proteins, amino acids, and carbohydrate compounds were adsorbed on the surface of CME-CuNPs [41], and these components are functionalized in reducing and stabilizing the CME-CuNPs. Moreover, CME contains secondary metabolite components such as phenolics, flavonoids, saponins, tannins, and terpenes. For this reason, we used CME in the biosynthesis of CME-CuNPs. Also, CME has wound-healing, antibacterial, and anti-inflammatory properties [42].

Our results show that MIC1, BAC1, and PSE5 are compatible with the conclusions of the morphological and biochemical characterization. Various studies have demonstrated that $M$. luteus, B. subtilis, and P. aeruginosa can be isolated from dental plaque and dental pulp $[43,44]$.

The present study's antimicrobial results document the high potential of CME-CuNPs with clindamycin against periodontal disease-causing microorganisms ( $M$. luteus, B subtilis, and P. aeruginosa) compared to CMECuNPs or clindamycin alone. Our results demonstrated the greater synergistic effect of the solution containing CME-CuNPs and clindamycin and anti-periodontal disease agents against tested clinical strains, because of its low MIC values. The antibacterial efficacy of some nanoparticles such as CME-CuNPs have been evaluated in previous studies and different mechanisms have been proposed for their effects [45]. The antibacterial efficacy of CME-CuNPs was related to its capability to distort the bacterial cell and cause multiple effects of reactive oxygen species (ROS) formation, and liberation of $\mathrm{Cu}$ ions, which lead to lipid peroxidation, protein oxidation, and DNA degradation in bacterial cells [46]. The inhibition of growth in selected bacteria and ROS formation was caused by electrostatic attraction between negatively charged bacterial cells and positively charged nanoparticles [47].

Moreover, clindamycin has a synergy effect on the antibacterial efficacy of the CME-CuNPs on tested bacteria. The reaction between clindamycin and CME-CuNPs led to synergism. The clindamycin molecule contains active groups like hydroxyl and amide, which react easily with CME-CuNPs by chelation [48]. The rationale for using drug combinations (CME-CuNPs with clindamycin) is the expectation that effective combinations might lower the incidence of bacterial resistance, reduce the host toxicity of the antimicrobial agents or enhance bactericidal activity $[44,49]$.

In conclusion, the CME-CuNPs prepared by biological synthesis using CME are more secure, cost-effective, and eco-friendly. The biological synthesis approach for CME-CuNPs has many advantages, such as being simple to use with commercial viability. The synergistic solution of clindamycin with CME-CuNPs displayed more significant antimicrobial activity compared to clindamycin or CME-CuNPs alone against selected bacteria, such as M. luteus, B. subtilis, and P. aeruginosa. Therefore, the obtained eco-friendly biosynthesized CME-CuNPs can be used for their enhanced efficacy in bio-dental fields for exploiting the existing antibiotics and other drugs and will result in the development of cost-effective treatments for infections and periodontal diseases in the future.

\section{Funding}

This work was funded by Deanship of Scientific Research, Taif University (research project No. 1/439/6084), Taif, Saudi Arabia.

\section{Acknowledgments}

We would like to express our gratitude to Deanship of Scientific Research, Taif University, Taif, Saudi Arabia for financial support under the research project number (1/439/6084). This study was approved by the Research Ethics Committee of Taif University, Taif, Saudi Arabia (No. 41-1107-00152). The authors declare that the funding bodies had no role in the design of the study, the collection, analysis, and interpretation of data, or in writing the manuscript.

\section{Conflict of Interest}

The authors have no financial conflicts of interest to declare. 


\section{References}

1. Global, regional, and national incidence, prevalence, and years lived with disability for 354 diseases and injuries for 195 countries and territories, 1990-2017: a systematic analysis for the Global Burden of Disease Study 2017. Lancet 392: 1789-8583.

2. Dias HB, Carrera ET, Bortolatto JF, De Andrade MF, De Souza Rastelli AN. 2016. LED and low-level laser therapy association in tooth bleaching using a novel low concentration $\mathrm{H}_{2} \mathrm{O}_{2} / \mathrm{N}$-doped $\mathrm{TiO}_{2}$ bleaching agent. Laser Phys. 26: 015602.

3. Petersen PE, Ogawa H. 2012. The global burden of periodontal disease: Towards integration with chronic disease prevention and control. Periodontol. 2000. 60: 15-39.

4. Chen X, Wu G, Feng Z, Dong Y, Zhou W, Li B, et al. 2016. Advanced biomaterials and their potential applications in the treatment of periodontal disease. Crit. Rev. Biotechnol. 36: 760-775.

5. Osorio R, Alfonso-Rodríguez CA, Medina-Castillo AL, Alaminos M, Toledano M. 2016. Bioactive polymeric nanoparticles for periodontal therapy. PLoS One 11: e 0166217.

6. Knight ET, Liu J, Seymour GJ, Faggion Jr. CM, Cullinan MP. 2016. Risk factors that may modify the innate and adaptive immune responses in periodontal diseases. Periodontol. 2000. 71:22-51.

7. Souto R, Silva-Boghossian CM, Colombo APV. 2014. Prevalence of Pseudomonas aeruginosa and Acinetobacter spp. in subgingival biofilm and saliva of subjects with chronic periodontal infection. Braz. J. Microbiol. 45: 495-501.

8. Viciani E, Montagnani F, Tordini G, Romano A, Salerni L, De Luca A, et al. 2017. Prevalence of M75 Streptococcus pyogenes strains harboring slaA gene in patients affected by pediatric obstructive sleep apnea syndrome in central Italy. Front. Microbiol. 8: 294.

9. Culotti A, Packman AI. 2014. Pseudomonas aeruginosa promotes Escherichia coli biofilm formation in nutrient-limited medium. PLoS One 9: e107186.

10. Colombo AP, Magalhaes CB, Hartenbach FA, do Souto RM, da Silva-Boghossian CM. 2016. Periodontal-disease-associated biofilm: a reservoir for pathogens of medical importance. Microb. Pathog. 94:27-34.

11. Sudiono J, Sandra F, Halim NS, Kadrianto TA, Melinia M. 2013. Bactericidal and cytotoxic effects of Erythrina fusca leaves aquadest extract. Dent. J. Majal. Kedokt. Gigi 46: 9-13.

12. Mah, TF. 2012. Biofilm-specific antibiotic resistance. Future Microbiol. 7: 1061-1072.

13. Victor T Noronha, Amauri J Paula, Gabriela Durán, Andre Galembeck, Karina Cogo-Müller, Michelle Franz-Montan. et al. 2017. Silver nanoparticles in dentistry. Dent. Mater. 33: 1110-1126.

14. Roshna T, Nandakumar K. 2012. Generalized aggressive periodontitis and its treatment options: case reports and review of the literature. Case Rep. Med. 2012: 535321.

15. Michaud DS, Fu Z, Shi J, Chung M. 2017. Periodontal disease, tooth loss, and cancer risk. Epidemiol. Rev. 39: 49-58.

16. Rieuwpassa IE, Achmad H, Rahmasari R. 2019. Effectiveness of clindamycin in treatment of Periodontitis. Indian J. Public Health Res. Dev. 10:1223.

17. Wyszogrodzka G, Marszałek B, Gil B, Doro zy'nski P. 2016. Metalorganic frameworks: mechanisms of antibacterial action and potential applications. Drug Discov. Today 21: 1009-1018.

18. D paz-Visurraga J, Daza C, Pozo C, Becerra A, von Plessing C, Garc $\varphi$ a 2012. A study on antibacterial alginate-stabilized copper nanoparticles by FT-IR and 2D-IR correlation spectroscopy. Int. J. Nanomed. 7:3597.

19. Holla G, Yeluri R, Munshi AK. 2012. Evaluation of minimum inhibitory and minimum bactericidal concentration of nano-silver base inorganic anti-microbial agent (Novaron) against Streptococcus mutans. Contemp. Clin. Dent. 3: 288-293.

20. Greenstein G, Tonetti M. 2000. The role of controlled drug delivery for periodontitis. The Research, Science and Therapy Committee of the American Academy of Periodontology. J. Periodontol. 71: 125-140.

21. Rajeshkumar S. 2016. Anticancer activity of eco-friendly gold nanoparticles against lung and liver cancer cells. J. Genet. Eng. Biotechnol. 14:195-202.

22. Podstawczyk D, Pawłowska A, Bastrzyk A, Czeryba M, Oszmia'nski J. 2019. Reactivity of (+)-catechin with copper (II) ions: the green synthesis of size-controlled Sub-10 nm copper nanoparticles. ACS Sustain. Chem. Eng. 7:17535-17543.

23. Molnár Z, Bódai V, Szakacs G, Erdélyi B, Fogarassy Z, Sáfrán G, Varga T, et al. 2018. Green synthesis of gold nanoparticles by thermophilic filamentous fungi. Sci. Rep. 8: 3943.

24. Salem MZM, Elansary HO, Ali HM, El-Settawy AA, Elshikh MS, Abdel-Salam EM, et al. 2018. Bioactivity of essential oils extracted from Cupressus macrocarpa branchlets and Corymbia citriodora leaves grown in Egypt. BMC Complement Altern. Med. 18: 23.

25. Enan ET, Ashour AA, Basha S, Felemban NH, Gad El-Rab SMF. 2021. Antimicrobial activity of biosynthesized silver nanoparticles, Amoxicillin and glass-ionomer cement against Streptococcus mutans and Staphylococcus aureus. Nanotechnology 32: 215101 (11pp).

26. Yaqub A, Malkani N, Shabbir A, Ditta S, Tanvir F, Ali S, et al. 2020. Novel biosynthesis of copper nanoparticles using Zingiber and Allium sp. with synergic effect of doxycycline for anticancer and bactericidal activity. Curr. Microbiol. 77:2287-2299.

27. Gad El-Rab SMF, Abo-Amer AE, Asiri AM. 2020. Biogenic synthesis of ZnO nanoparticles and its potential use as antimicrobial agent against multidrug-resistant pathogens. Curr. Microbiol. 77:1767-1779.

28. Gad El-Rab SMF, Halawani EM, Hassan AM. 2018. Formulation of ceftriaxone conjugated gold nanoparticles and their medical applications against extended-spectrum $\beta$-Lactamase producing bacteria and breast cancer. World J. Microbiol. Biotechnol. 28: 1563-1572.

29. Dubey SP, Lahtinen M, Sillanpaa M. 2010. Tansy fruit mediated greener synthesis of silver and gold nanoparticles. Process Biochem. 45: $1065-1071$

30. Halawani EM, Hassan AM, Gad El-Rab SMF. 2020. Nanoformulation of biogenic cefotaxime-conjugated-silver nanoparticles for enhanced antibacterial efficacy against multidrug-resistant bacteria and anticancer studies. Int. J. Nanomed. 5: 1889-1901.

31. John G. Holt PhD. 1994. Bergey's manual of determinative bacteriology $9^{\text {th }}$ edn (Baltimore, Maryland: Williams \& Wilkins) pp. 20: 527-558.

32. MaccFadin JK. 2000. Biochemical test for identification of medical bacteria 3rd edn (New York: Lippincott Williams and Winkins, AwolterKlumer Company. Philadelphia Baltimore).

33. Kanmani P, Lim ST. 2013. Synthesis and characterization of pullulan-mediated silver nanoparticles and its antimicrobial activities. Carbohydr. Polym. 12: 421-428.

34. Iskandarsyah NH, Rosana Y. 2020. Sinergicity test of silver nanoparticles and clindamycin against Staphylococcus aureus. Int. J. Res. Pharm. Sci. 11: 1192-1198.

35. Wu S, Rajeshkumar S, Madasamy M, Mahendran V. 2020. Green synthesis of copper nanoparticles using Cissus vitiginea and its antioxidant and antibacterial activity against urinary tract infection pathogens. Artif. Cells Nanomed. Biotechnol. 48: 1153-1158.

36. Joseph AT, Prakash P, Narvi S. 2016. Phytofabrication and Characterization of copper nanoparticles using Allium sativum and its antibacterial activity. Int. J. Sci. Eng. Technol. 4: 463-472.

37. Sengan M, Veerappan A. 2019. $N$-myristoyltaurine capped copper nanoparticles for selective colorimetric detection of $\mathrm{Hg}^{2+}$ in wastewater and as effective chemocatalyst for organic dye degradation. Microchem. J. 148: 1-9.

38. Suarez-Cerda J, Espinoza-Gómez H, Alonso-Núñez G, et al. 2017. A green synthesis of copper nanoparticles using native cyclodextrins as stabilizing agents. J. Saudi Chem. Soc. 21:341-348. 
39. Fan D, Zhou Q, Lv X, Jing J, Ye Z, Shao S, Xie J. 2018. Synthesis, thermal conductivity and anti-oxidation properties of copper nanoparticles encapsulated within few-layer h-BN. Ceram. Int. 44:1205-1208.

40. Dutta D, Phukan A, Dutta DK. 2018. Nanoporous montmorillonite clay stabilized copper nanoparticles: efficient and reusable catalyst for oxidation of alcohols. Mol. Catal. 451: 178-185.

41. Gurunathan S, Kalishwaralal K, Vaidyanathan R, Venkataraman D, Pandian SR, Muniyandi J, et al. 2009. Biosynthesis, purification and characterization of silver nanoparticles using Escherichia coli. Colloids Surf. B 74: 328-335.

42. Harraz FM, Hammoda, El-Hawiet A, Radwan MM, Wanas, Eid AME, ISohly MA. 2018. From natural product research chemical constituents, Antibacterial and Acetylcholine esterase inhibitory activity of Cupressus macrocarpa leaves. Nat. Prod. Res. 34: 816822

43. Fuloria NK, Fuloria S, Chia KY, Karupiah S, Sathasivam K. 2019. Response of green synthesized drug blended silver nanoparticles against periodontal disease triggering pathogenic microbiota. J. Appl. Biol. Biotechnol. 7: 46-56.

44. Emmanuel R, Palanisamy S, Chen S, Chelladurai K, Padmavathy S, Saravanan M, et al. 2015. Antimicrobial efficacy of green synthesized drug blended silver nanoparticles against dental caries and periodontal disease-causing microorganisms. Mater. Sci. Eng. C 56: 374-379.

45. Li Y, Zhang W, Niu J, Chen Y. 2012. Mechanism of photogenerated reactive oxygen species and correlation with the antibacterial properties of engineered metal-oxide nanoparticles. ACS Nano 6: 5164-5173.

46. Chatterjee AK, Chakraborty R, Basu T. 2014. Mechanism of antibacterial activity of copper nanoparticles. Nanotechnology 25: 135101

47. Arumugam A, Karthikeyan C, Haja Hameed AS, Gopinath K, Gowri S, Karthika V. 2015. Synthesis of cerium oxide nanoparticles using Gloriosa superba L. leaf extract and their structural, optical and antibacterial properties. Mater. Sci. Eng. C. 49: 408-415.

48. Mandava K, Kadimcharla K, Keesara NR, Sumayya NF, Prathyusha B, Batchu UR. 2017. Green synthesis of stable copper nanoparticles and synergistic activity with antibiotics. Indian J. Pharm. Sci. 79: 695-700.

49. Gad El-RabSMF, Halawani EM, Alzahrani SSS. 2021. Biosynthesis of silver nano-drug using Juniperus excelsa and its synergistic antibacterial activity against multidrug-resistant bacteria for wound dressing applications. 3 Biotech 11: 255.

50. Patel BH, Channiwala MZ, Chaudhari SB, Mandot AA. 2016. Biosynthesis of copper nanoparticles; its characterization and efficacy against human pathogenic bacterium. J. Environ. Chem. Eng. 4: 2163-2169.

51. Hassanien R, Husein DZ, Al-Hakkani MF. 2018. Biosynthesis of copper nanoparticles using aqueous Tilia extract: antimicrobial and anticancer activities. Heliyon 4: e01077.

52. Rajeshkumar S, Menon S, Kumar SV, Tambuwala MM, Bakshi H A, Mehta M, et al. 2019. Antibacterial and antioxidant potential of biosynthesized copper nanoparticles mediated through Cissus arnotiana plant extract. J. Photochem. Photobiol. B. 197: 111531.

53. Covarrubias C, Trepiana D, Corral C. 2018. Synthesis of hybrid copper-chitosan nanoparticles with antibacterial activity against cariogenic Streptococcus mutans. Dent. Mater. J. 37: 379-384.

54. Mardones J, Gómez ML, Díaz C, Galleguillos C, Covarrubias C. 2018. In vitro antibacterial properties of copper nanoparticles as endodontic medicament against Enterococcus faecalis. J. Dent. Oral Disord. 4: 1107. 